Supplement of Geosci. Model Dev., 11, 2503-2523, 2018

https://doi.org/10.5194/gmd-11-2503-2018-supplement

(C) Author(s) 2018. This work is distributed under

the Creative Commons Attribution 4.0 License.

(c) (1)

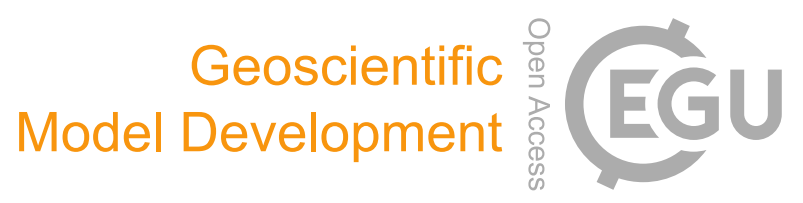

Supplement of

\title{
A conservative reconstruction scheme for the interpolation of extensive quantities in the Lagrangian particle dispersion model FLEXPART
}

S. Hittmeir et al.

Correspondence to: Sabine Hittmeir (sabine.hittmeir@univie.ac.at)

The copyright of individual parts of the supplement might differ from the CC BY 4.0 License. 


\section{Contents}

1 Python Code of the Interpolation Algorithms 1

1.1 Interpolation algorithm IA $1 \ldots \ldots \ldots \ldots \ldots \ldots \ldots \ldots \ldots$

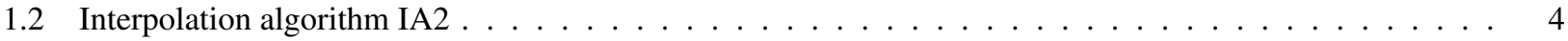

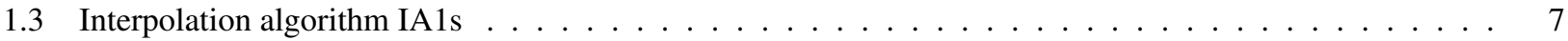

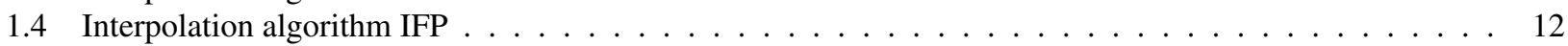

2 ECMWF MARS Extraction Code $\quad 13$

2.1 MARS retrieval for 3 -hourly data $\ldots \ldots \ldots \ldots \ldots \ldots \ldots \ldots \ldots$

2.2 MARS retrieval for 1 -hourly data $\ldots \ldots \ldots \ldots \ldots \ldots \ldots \ldots$

2.3 Splitting the GRIB files $\ldots \ldots \ldots \ldots \ldots \ldots \ldots \ldots \ldots$

\section{S1. Python Code of the Interpolation Algorithms}

\section{S1.1. Interpolation algorithm IA1}

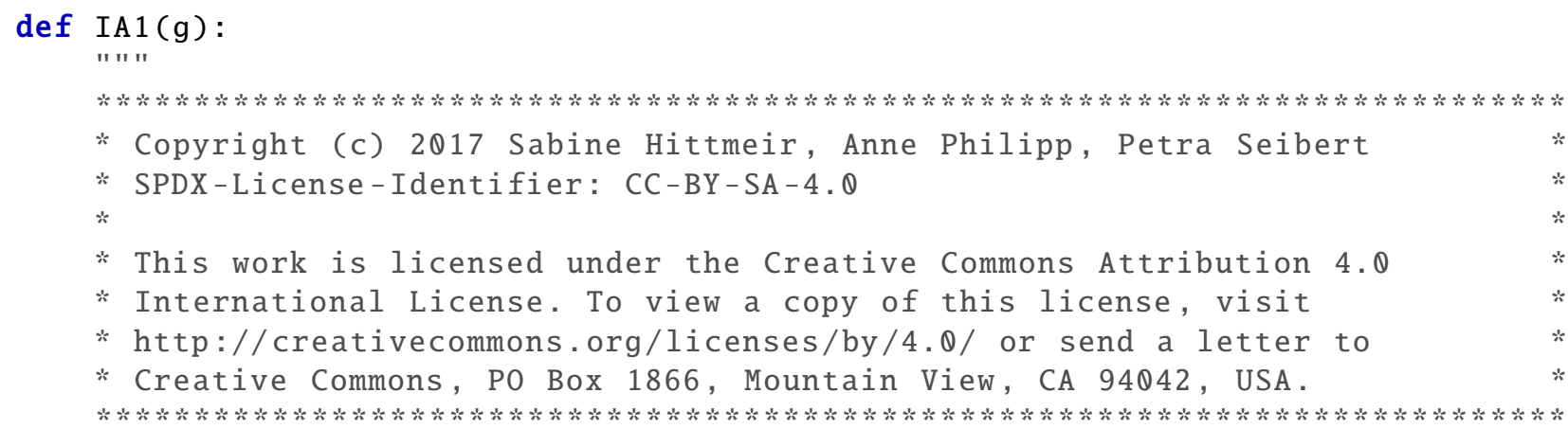



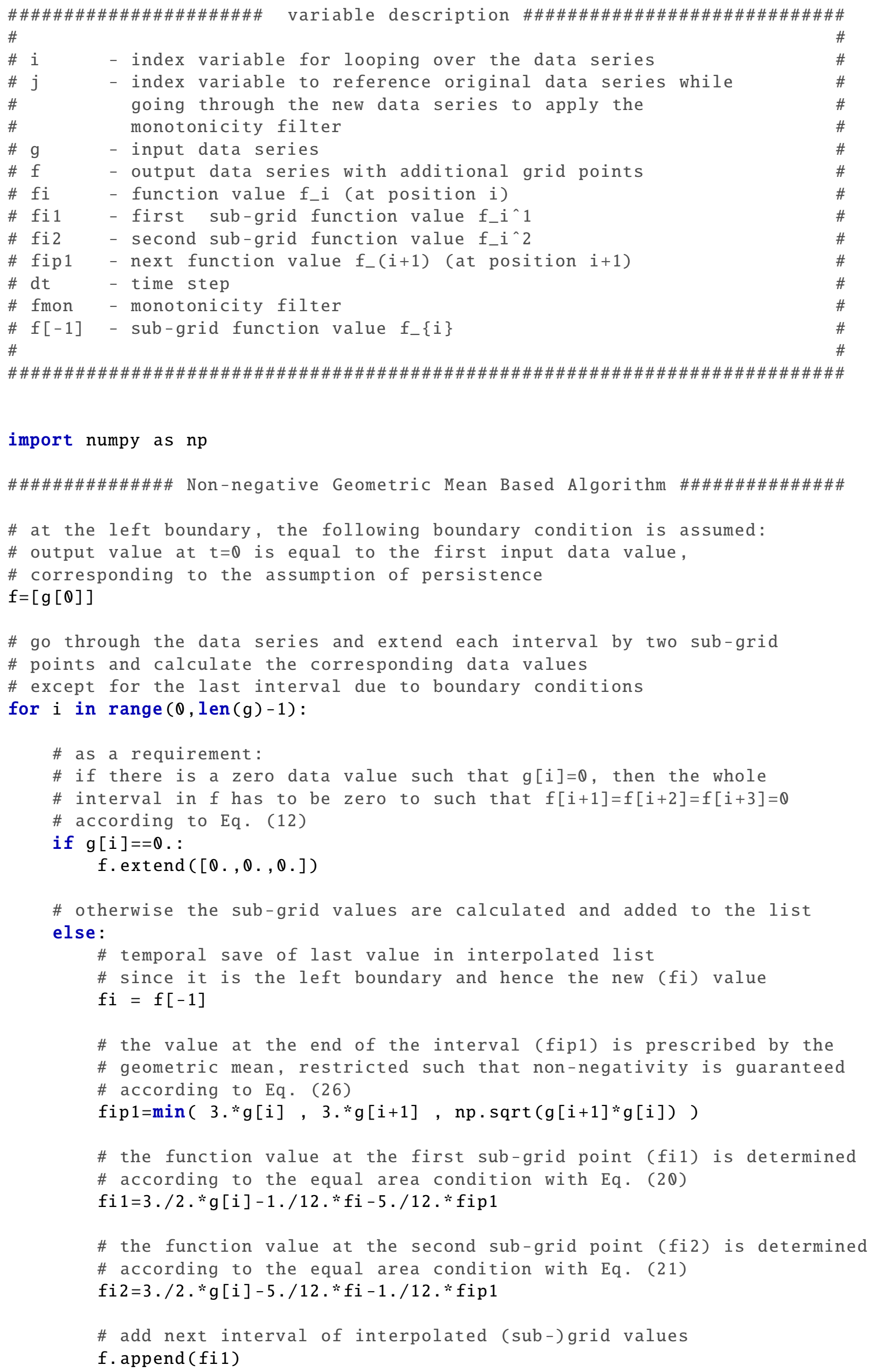


\section{f. append ( $f i 2)$}

f. append (fip1)

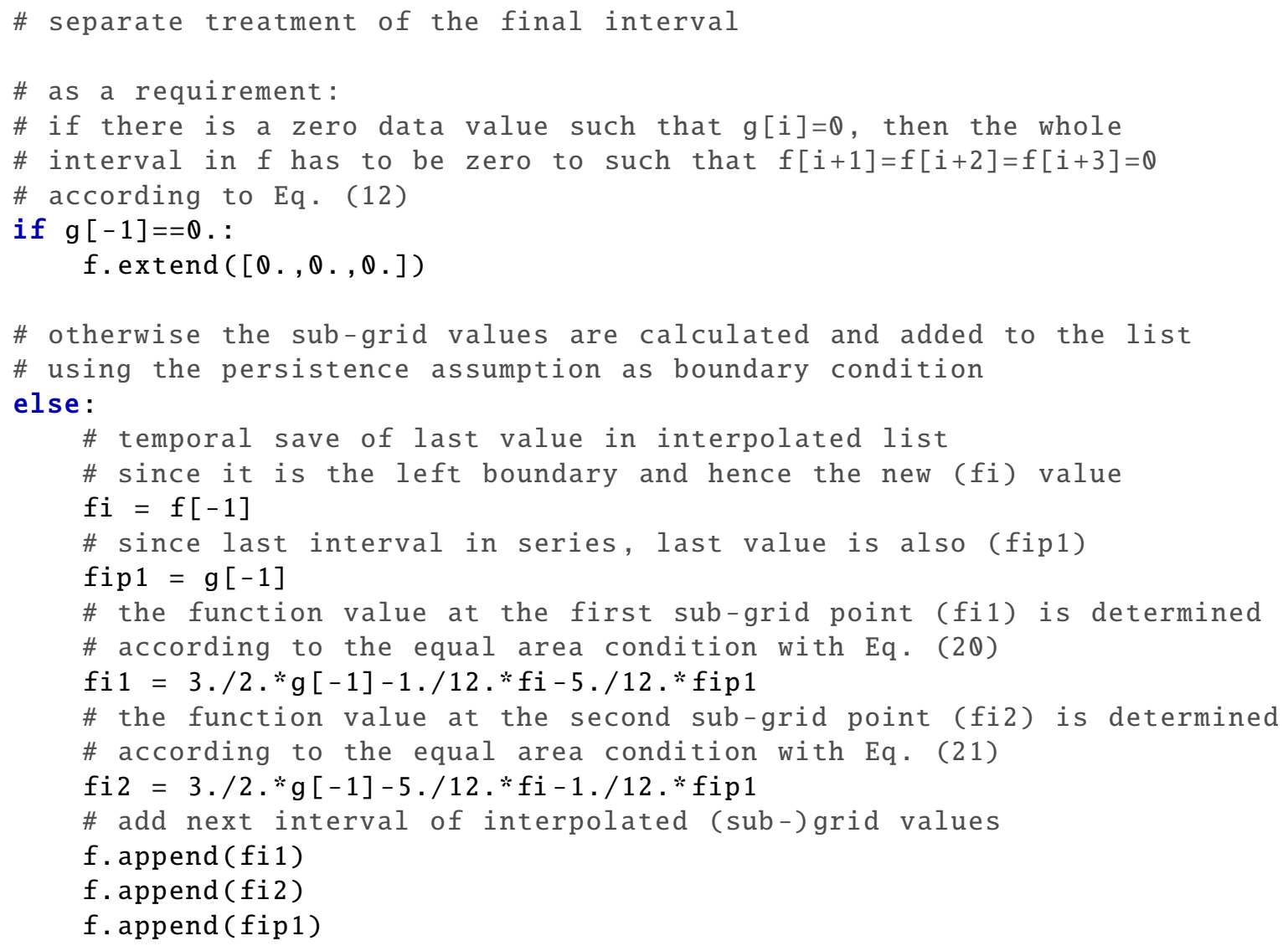

\#\#\#\#\#\#\#\#\#\#\#\#\#\#\#\#\#\#\#\# Monotonicity Filter \#\#\#\#\#\#\#\#\#\#\#\#\#\#\#\#\#\#\#\#\#\#\#

for $i$ in range $(3, \operatorname{len}(f)-4,3)$ :

$j=\operatorname{int}(i / 3)-$.

\# otherwise, if the monotonicity property is violated (test Eq.

(27))

\# we replace the interpolated function values according to the

\# specifications in the actual and consecutive interval

if $\quad n p \cdot \operatorname{sign}(f[i-1]-f[i-2]) * n p \cdot \operatorname{sign}(f[i]-f[i-1])==-1$ । and $n p \cdot \operatorname{sign}(f[i+1]-f[i]) * n p \cdot \operatorname{sign}(f[i]-f[i-1])==-1 \backslash$

and $n p . \operatorname{sign}(f[i+2]-f[i+1]) * n p \cdot \operatorname{sign}(f[i+1]-f[i])==-1$ :

\# the monotonicity filter corrects the value at (fip1) by \# substituting (fip1) with (fmon), see Eq. (28), (29) and (30)

fmon $=\min (3 \cdot * \mathrm{~g}[\mathrm{j}], \backslash$ 3. $* g[j+1], ।$

return $f$ 


\section{S1.2. Interpolation algorithm IA2}

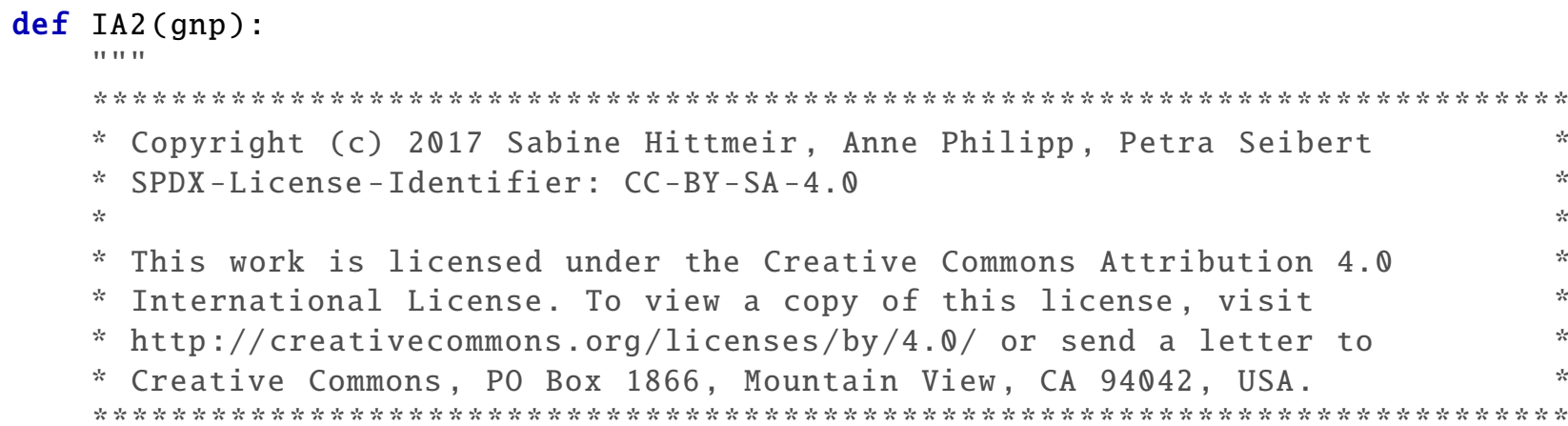

aDescription

The input to this python function is a $1 \mathrm{~d}$ array of data passed as parameter $\mathrm{g}$. If $\mathrm{g}$ has $\mathrm{n}$ elements, it returns an array of size $(\mathrm{n}-1) * 3+1$ as the result for IA2 where additional supporting points have been added in each interval.

A reconstruction by linear interpolation between these points is conservative with respect to the input (where we consider each list element to represent a mean value for the preceding interval) and positive definite. The function includes a monotonicity filter which is already incorporated in the reconstruction algorithm by making an educated guess for the $f_{-}\{i+2\}$ function value.

For more information see article:

Hittmeir, S.; Philipp, A.; Seibert, P. (2018): A conservative reconstruction scheme for the interpolation of extensive quantities in the Lagrangian particle dispersion model FLEXPART.

Geoscientific Model Development

@Input

gnp: numpy array, shape(nt), float

Original data series which will be interpolated.

nt is the length of the time series.

@Return

$f:$ list of float values

The reconstructed input data series with the two additional sub-grid points for each interval of the input data. 


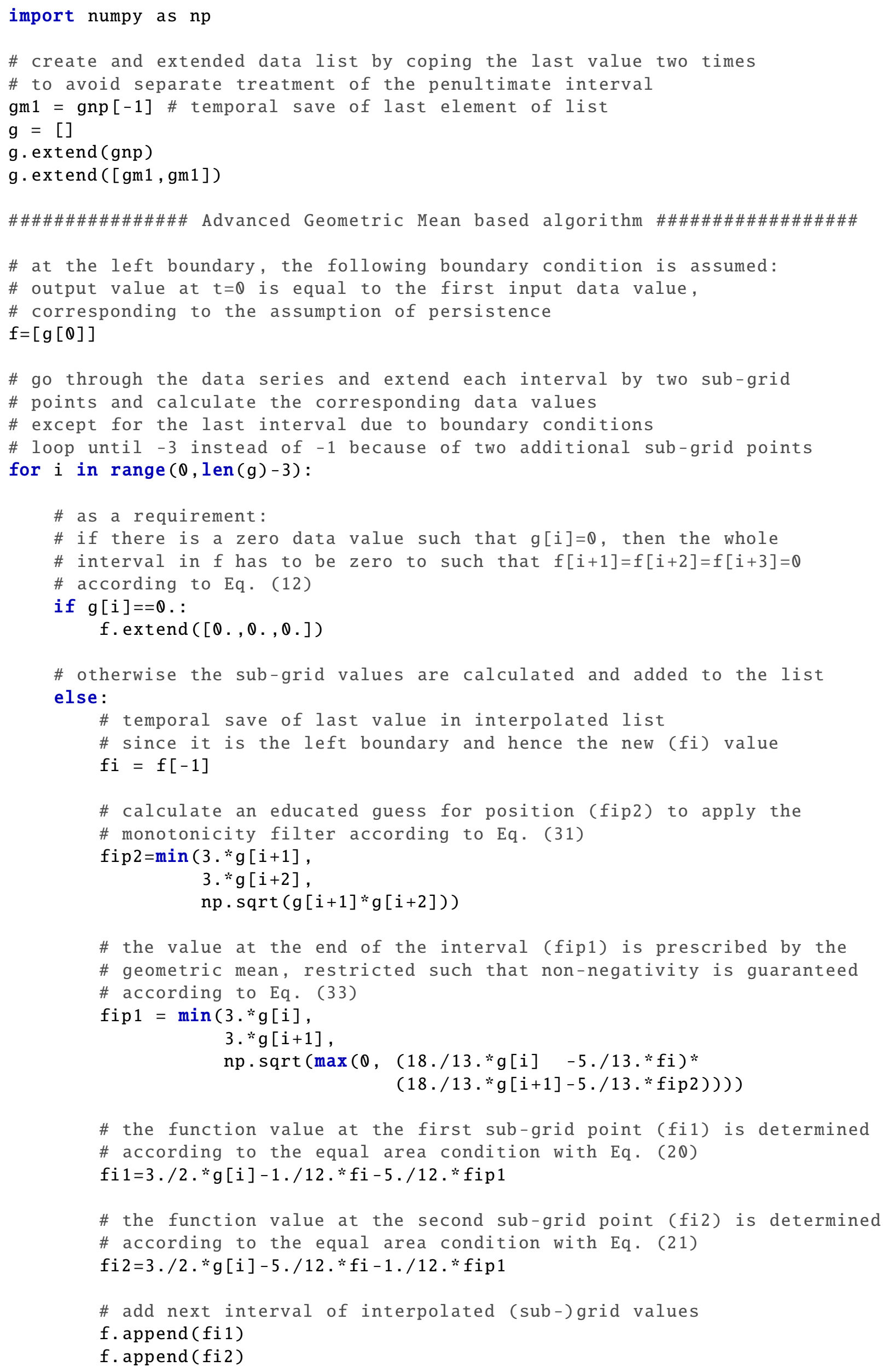




\section{f. append (fip1)}

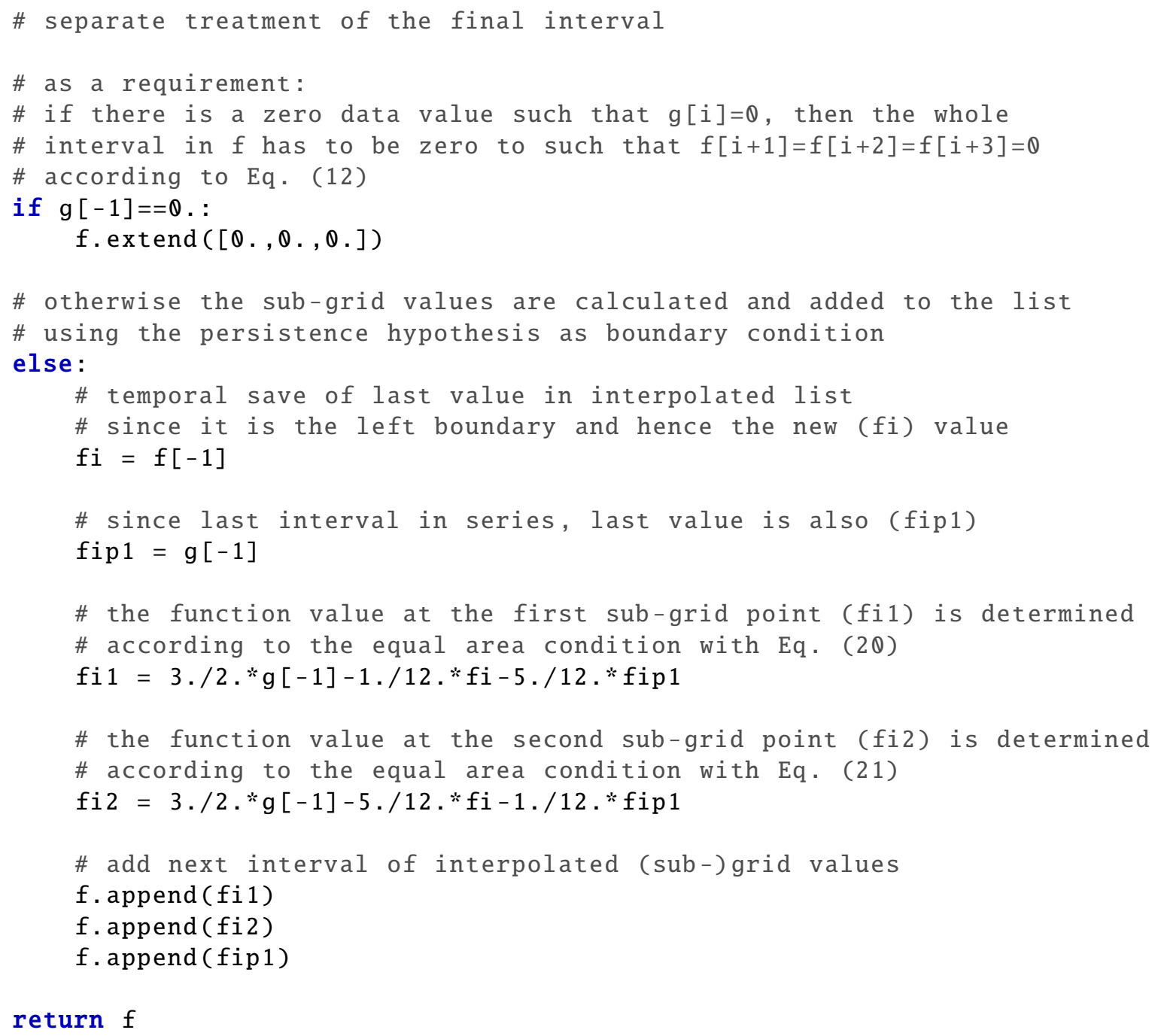

return $f$ 


\section{S1.3. Interpolation algorithm IA1s}

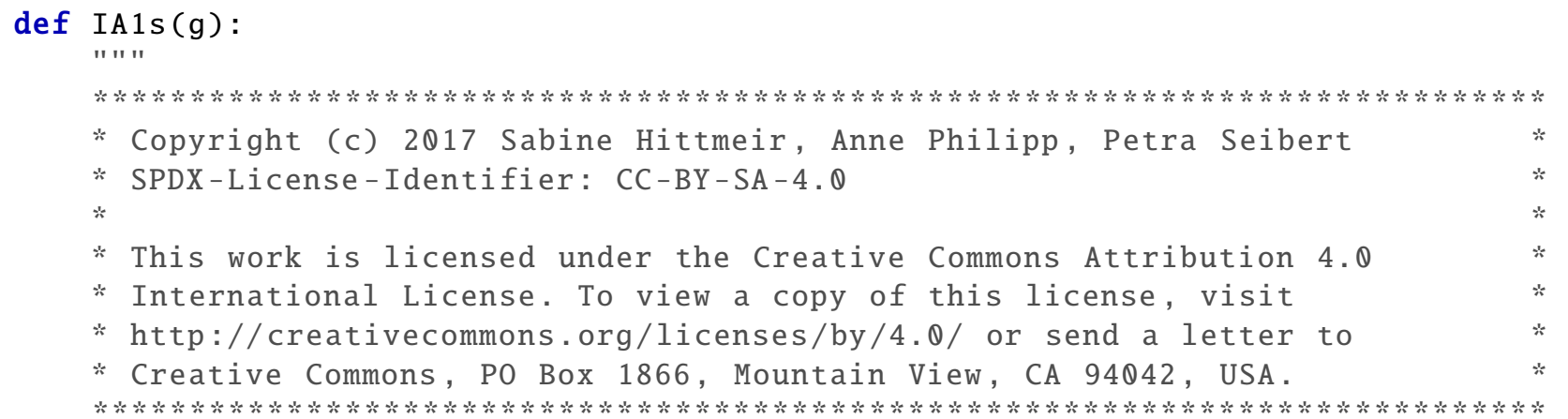

aDescription

The input to this python function is a $1 \mathrm{~d}$ array of data passed as parameter $\mathrm{g}$. If $\mathrm{g}$ has $\mathrm{n}$ elements, it returns an array of size $(n-1) * 3+1$ as the result for IA1s where additional supporting points have been added in each interval.

A reconstruction by linear interpolation between these points is conservative with respect to the input (where we consider each list element to represent a mean value for the preceding interval) and positive definite. The function includes a monotonicity filter applied directly in the first sweep.

For more information see article:

Hittmeir, S.; Philipp, A.; Seibert, P. (2018): A conservative reconstruction scheme for the interpolation of extensive quantities in the Lagrangian particle dispersion model FLEXPART.

Geoscientific Model Development

aInput

g: numpy array, shape(nt), float

Original data series which will be interpolated.

$\mathrm{nt}$ is the length of the time series.

@Return

$f:$ list of float values

The reconstructed input data series with the two additional

sub-grid points for each interval of the input data.

\#\#\#\#\#\#\#\#\#\#\#\#\#\#\#\#\#\# variable description \#\#\#\#\#\#\#\#\#\#\#\#\#\#\#\#\#\#\#\#\#\#\#\# $\#$

\# i - index variable for looping over the data series 


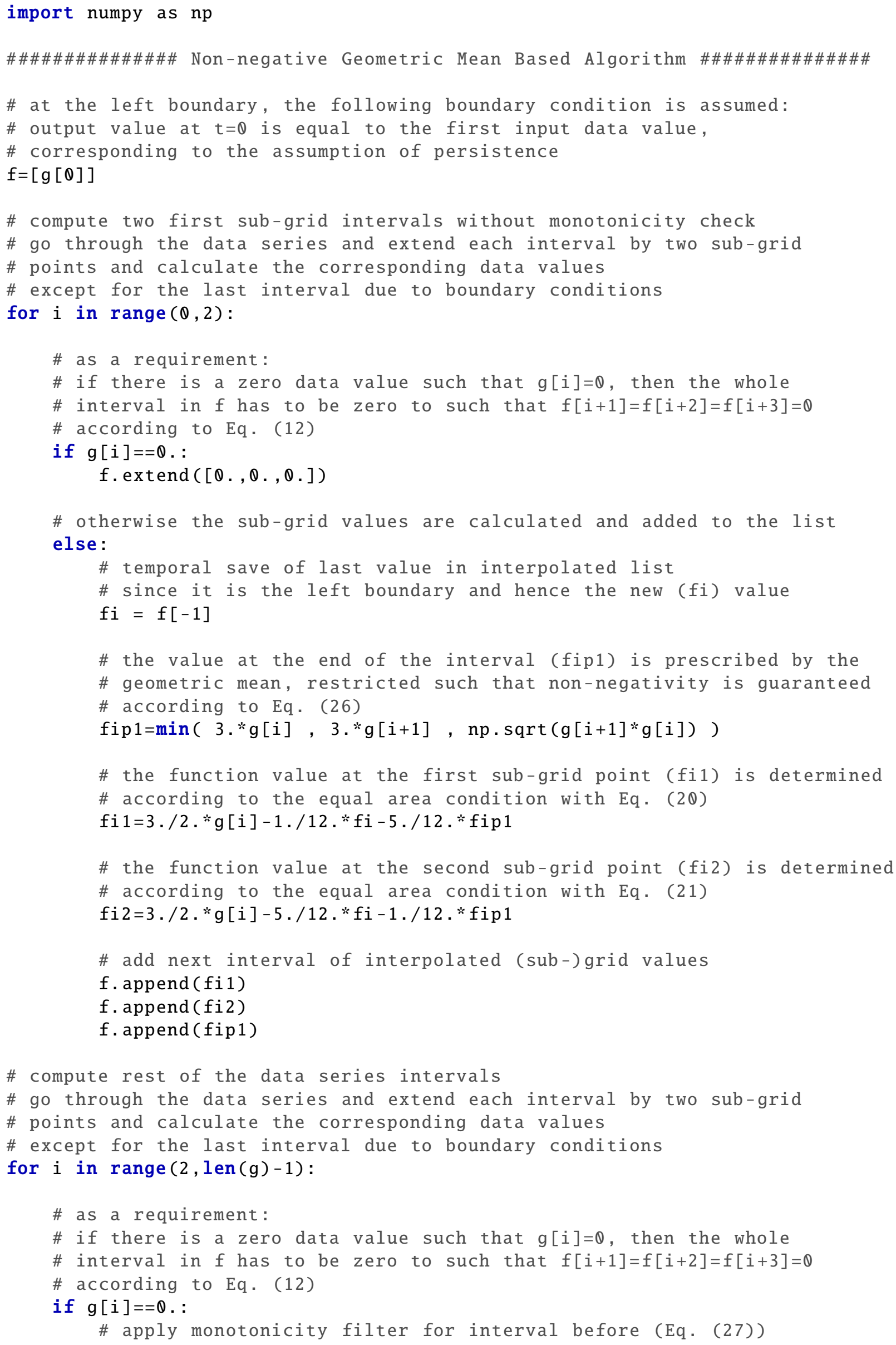


\# check if there is "M" or "W" shape

if $\quad n p . \operatorname{sign}(f[-5]-f[-6]) * n p \cdot \operatorname{sign}(f[-4]-f[-5])==-1 \backslash$

and $n p \cdot \operatorname{sign}(f[-4]-f[-5]) * n p \cdot \operatorname{sign}(f[-3]-f[-4])==-1 \backslash$

and $n p . \operatorname{sign}(f[-3]-f[-4]) * n p \cdot \operatorname{sign}(f[-2]-f[-3])==-1$ :

\# the monotonicity filter corrects the value at (fim1) by

\# substituting (fim1) with (fmon), see Eq. (28), (29) and (30)

fmon $=\min (3 \cdot * \mathrm{~g}[\mathrm{i}-2], \backslash$

3. *g $[i-1], ।$

$n p . \operatorname{sqrt}(\max (\theta,(18 . / 13 . * g[i-2]-5 . / 13 . * f[-7]) *$

$(18 . / 13 . * \mathrm{~g}[\mathrm{i}-1]-5 . / 13 . * \mathrm{f}[-1]))))$

\# re-computation of the sub-grid interval values while the

\# interval boundaries ( $f i$ ) and (fip2) remains unchanged

\# see $\mathrm{Eq} .(20)$ and (21)

$f[-6]=3 . / 2 . * g[i-2]-1 . / 12 * f[-7]-5 . / 12 . * f m o n$

$f[-5]=3 . / 2 . * g[i-2]-5 . / 12 . * f[-7]-1 . / 12 . *$ fmon

$\mathrm{f}[-4]=\mathrm{fmon}$

$f[-3]=3 . / 2 * \mathrm{~g}[\mathrm{i}-1]-1 . / 12 . * \mathrm{fmon}-5 . / 12 * \mathrm{f}[-1]$

$f[-2]=3 . / 2 * \mathrm{~g}[\mathrm{i}-1]-5 . / 12 . * \mathrm{fmon}-1 . / 12 . * \mathrm{f}[-1]$

f.extend $([0 ., 0 ., 0]$.

\# otherwise the sub-grid values are calculated and added to the list else:

\# temporal save of last value in interpolated list

\# since it is the left boundary and hence the new (fi) value

$f i=f[-1]$

\# the value at the end of the interval (fip1) is prescribed by the

\# geometric mean, restricted such that non-negativity is guaranteed

\# according to Eq. (26)

fip $1=\min (3 . * g[i], 3 . * g[i+1], \operatorname{np} \cdot \operatorname{sqrt}(g[i+1] * g[i]))$

\# the function value at the first sub-grid point (fi1) is determined

\# according to the equal area condition with Eq. (20)

fi $1=3 . / 2 * g[i]-1 . / 12 *$ fi $-5 . / 12 . * f i p 1$

\# the function value at the second sub-grid point (fi2) is determined

\# according to the equal area condition with Eq. (21)

$\mathrm{fi} 2=3 . / 2 . * \mathrm{~g}[\mathrm{i}]-5 . / 12 . * \mathrm{fi}-1 . / 12 . * \mathrm{fip} 1$

\# apply monotonicity filter for interval before (Eq. (27))

\# check if there is "M" or "W" shape

if $\quad n p . \operatorname{sign}(f[-5]-f[-6]) * n p \cdot \operatorname{sign}(f[-4]-f[-5])==-1 \backslash$

and $n p \cdot \operatorname{sign}(f[-4]-f[-5]) * n p \cdot \operatorname{sign}(f[-3]-f[-4])=-1 \backslash$

and $n p . \operatorname{sign}(f[-3]-f[-4]) * n p . \operatorname{sign}(f[-2]-f[-3])==-1$ :

\# the monotonicity filter corrects the value at (fim1) by

\# substituting ( $f$ im1) with fmon, see Eq. (28), (29) and (30)

fmon $=\min (3 \cdot * \mathrm{~g}[i-2], \backslash$

3. *g $[i-1]$,

$n p . \operatorname{sqrt}(\max (\theta,(18 . / 13 . * \mathrm{~g}[\mathrm{i}-2]-5 . / 13 . * f[-7]) *$

$(18 . / 13 . * g[i-1]-5 . / 13 . * f[-1]))))$

\# re-computation of the sub-grid interval values while the

\# interval boundaries (fi) and (fip2) remains unchanged

\# see Eq. (20) and (21)

$f[-6]=3 . / 2 . * g[i-2]-1 . / 12 * * f[-7]-5 . / 12 . * f m o n$

$f[-5]=3 . / 2 . * g[i-2]-5 . / 12 . * f[-7]-1 . / 12 . *$ fmon

$f[-4]=$ fmon 


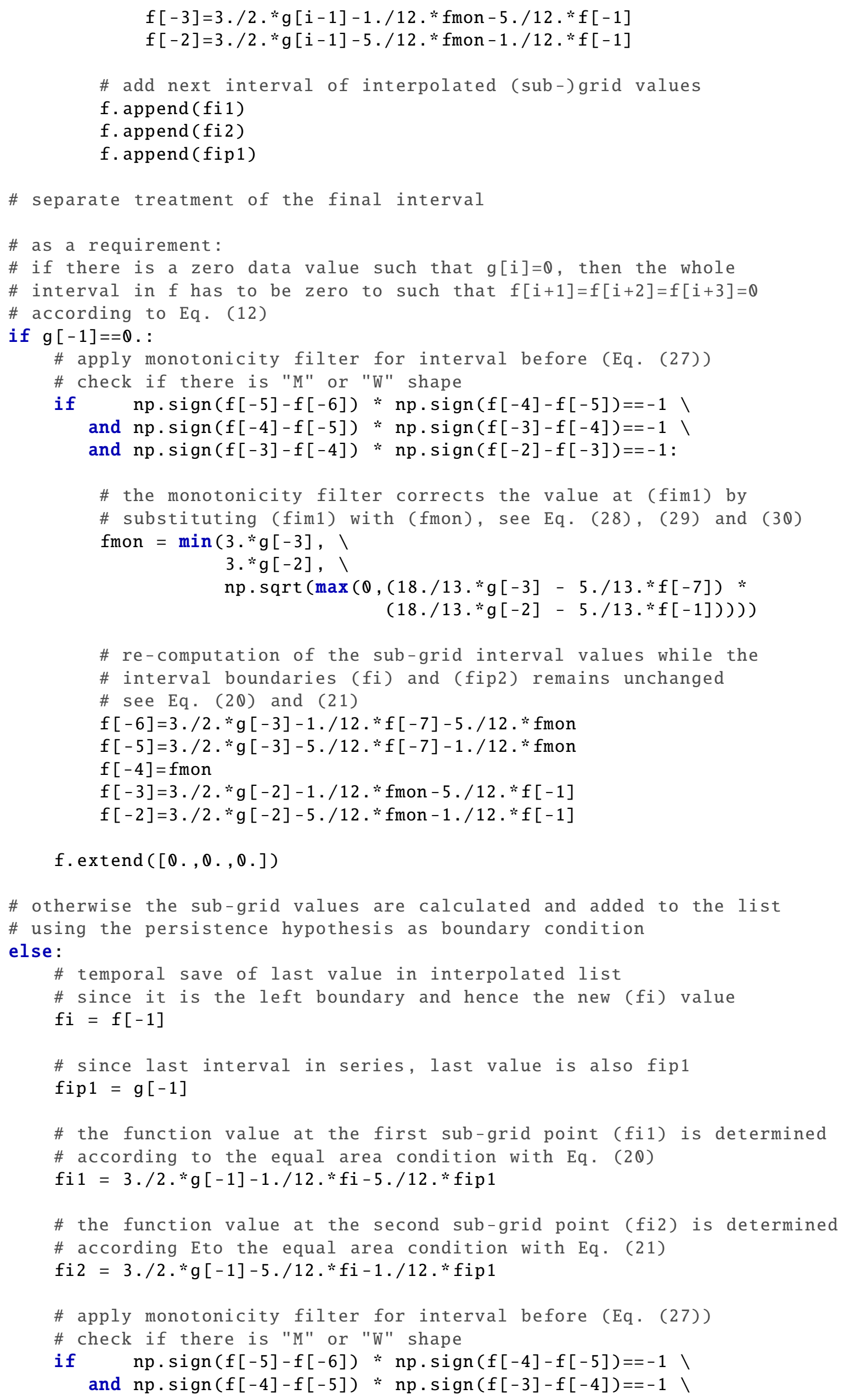


and $n p \cdot \operatorname{sign}(f[-3]-f[-4]) * n p \cdot \operatorname{sign}(f[-2]-f[-3])==-1$ :

\# the monotonicity filter corrects the value at (fim1) by \# substituting (fim1) with (fmon), see Eq. (28), (29) and (30) fmon $=\min (3 . * \mathrm{~g}[-3]$, $)$

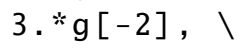

\# add next interval of interpolated (sub-)grid values

$f$. append ( $f i 1$ )

f. append ( $f i 2$ )

f. append (fip1)

return $f$ 


\section{S1.4. Interpolation algorithm IFP}

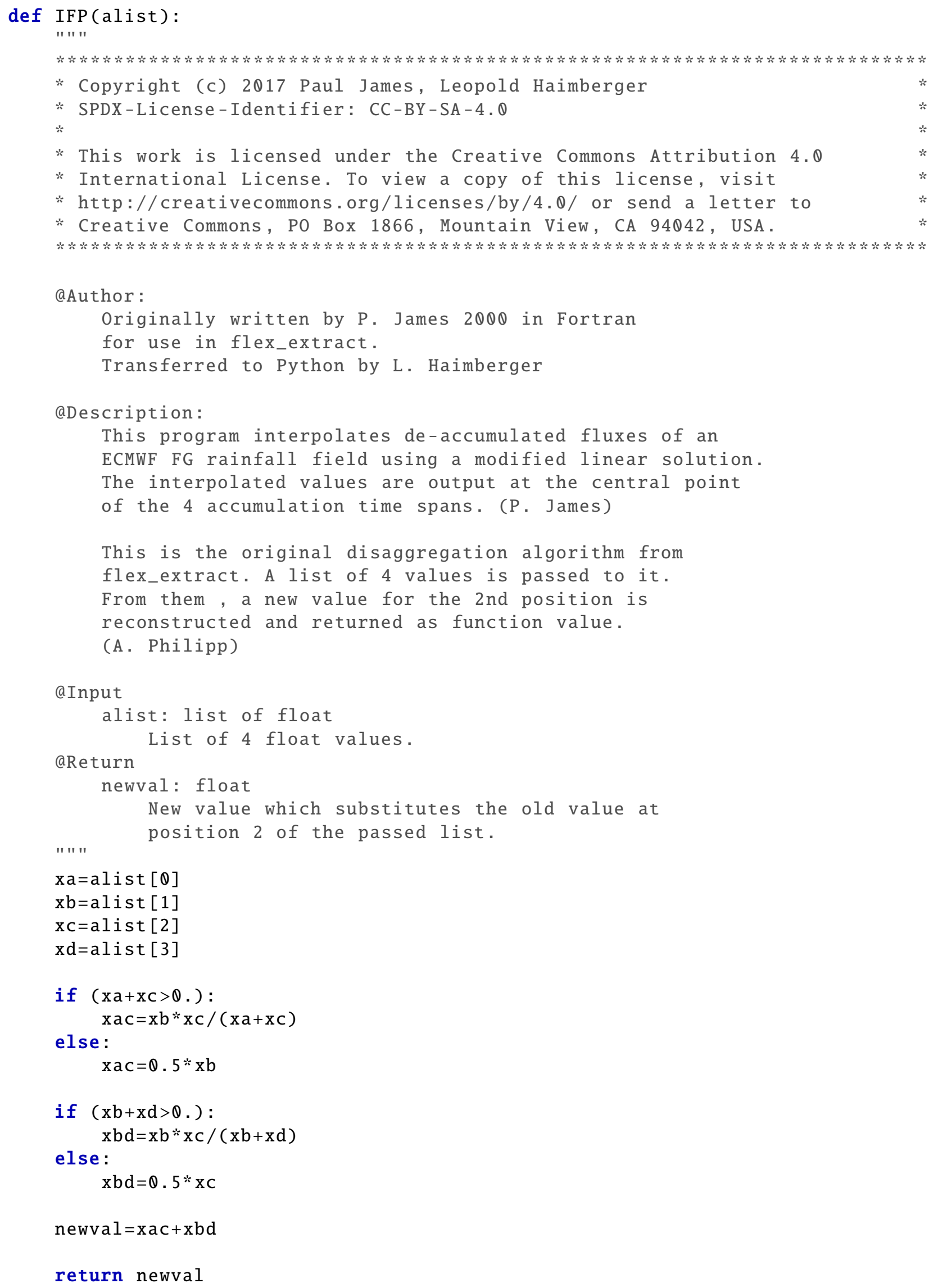




\section{S2. ECMWF MARS Extraction Code}

The following code can be used to retrieve the data used from ECMWF's MARS archive. Note that interactive access to ecaccess/ecgate is needed for this form of the code, and for the access to the operational data streams. It should not be too difficult, however, to modify this code in a way that it can be used to retrieve reanalysis data through the python API interface, an option that is available to the public after registration.

The 1-hourly and the 3-hourly data sets are extracted separately. Furthermore, precipitation data are not analysed parameters, therefore only forecasts can be used. We use the forecasts based on the 00 UTC analysis for the data between 01 and 12 UTC, and forecasts based on the 12 UTC analysis for the data between 12 and 24 UTC (00 UTC next day). A retrieval consists of the introductory code word retrieve and the parameters which specify the data. Parameter from the first retrieval not specified again with subsequent retrieve commands remain valid. Precipitation data for the 1st of January, 00 UTC, need to be fetched separately. Data are retrieved as GRIB files. The sample code below extracts convective and large-scale precipitation at once.

\section{S2.1. MARS retrieval for 3-hourly data}

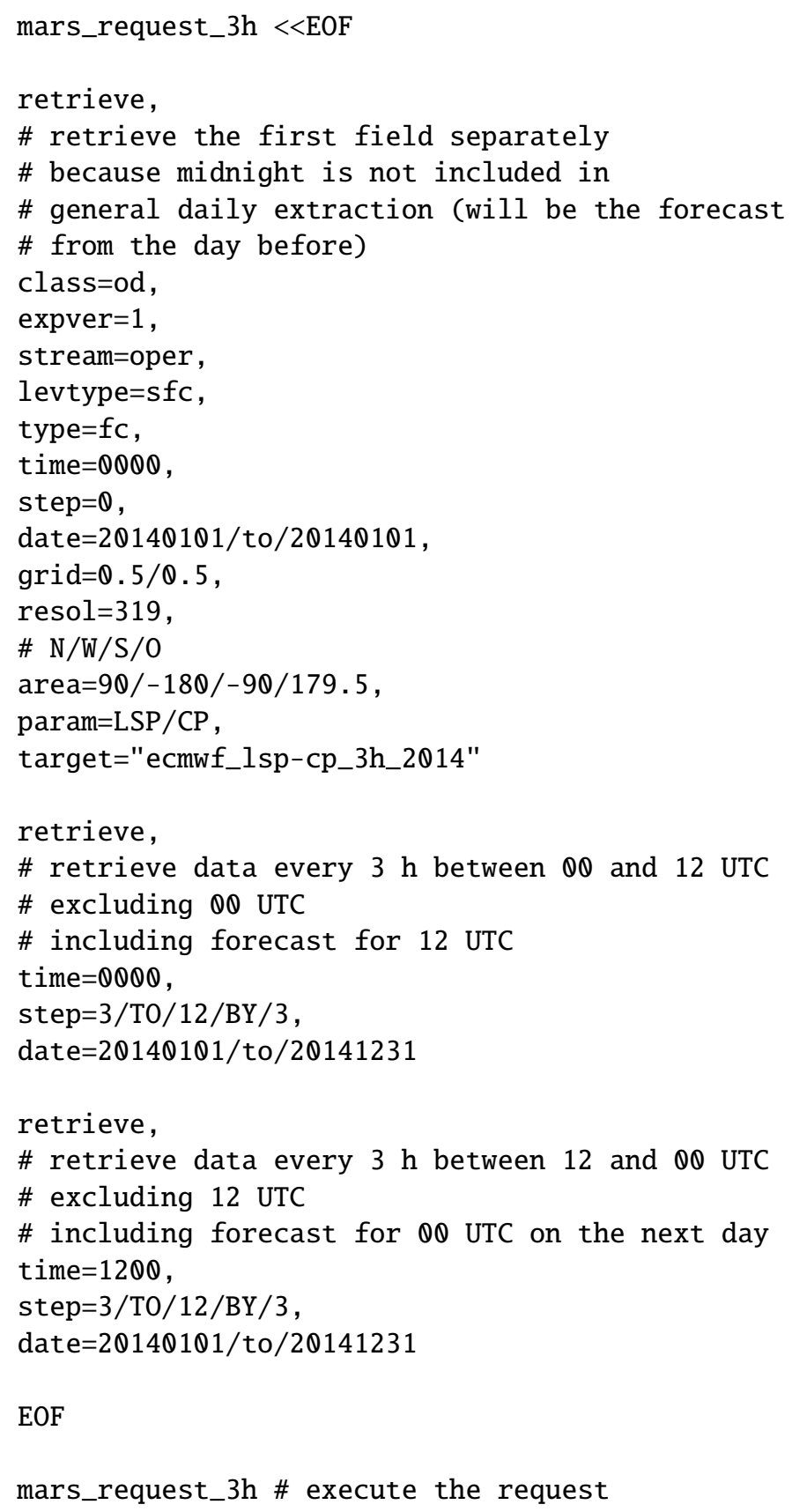




\section{S2.2. MARS retrieval for 1-hourly data}

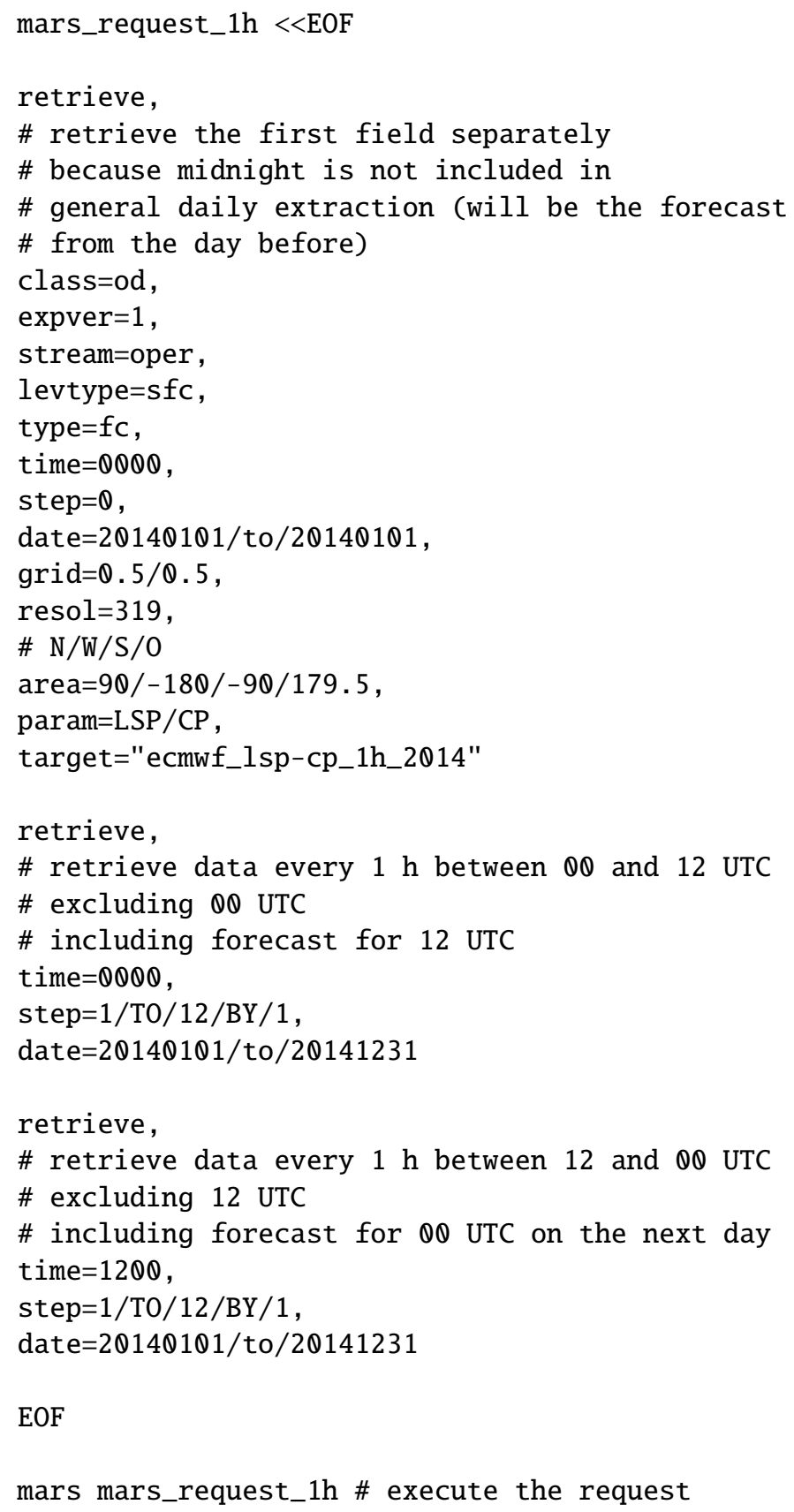

\section{S2.3. Splitting the GRIB files}

The code provided above collects the data in a single big GRIB file. In order to split it into daily files, create a file gfilter containing the following lines:

\# gribfilter for splitting data into daily files

\# change $<\mathrm{STEP}>$ to $1 \mathrm{~h}$ for 1 -hourly $3 \mathrm{~h}$ for the 3 -hourly data file

write "ecmwf_lsp-cp_<STEP>_[dataDate]";

and execute

grib_filter gfilter <infilename>.grib

$<$ STEP $>$ and $<$ infilename $>$ have to be replaced by appropriate values. 